\title{
An Analysis of Voice over Internet Protocol in Wireless Mesh Networks
}

\author{
Mohammad Tariq Meeran \\ Faculty of Computer Science \\ Kabul University \\ Kabul, Afghanistan \\ tariqmeeran@ku.edu.af/meeran@tlu.ee
}

\author{
William D. Tucker \\ Department of Computer Science \\ University of the Western Cape \\ Private bag X17, Bellville 7535, South Africa \\ btucker@uwc.ac.za
}

\begin{abstract}
The paper focuses on analyzing the affects of wireless mesh networks with some mobile nodes on Voice over Internet Protocol service quality. Our interest is to examine this in simulation to learn how to better deploy voice services on such a network in a rural community. Wireless mesh networks' unique characteristics like multihop, node mobility, coverage, and medium usage cause quality of service issues for Voice over Internet Protocol implementations. This research considers three wireless mesh scenarios on 26 mesh nodes. In the first scenario all nodes are stationary. In the second, 10 nodes are mobile and 16 nodes are stationary. In a third scenario, all nodes are mobile. Nodes move at a walking speed of $1.3 \mathrm{~m}$ per second. The analysis and results show that while node mobility can increase packet loss, delay, jitter, Voice over Internet Protocol implementations in wireless mesh networks can be successful if there is no background traffic. We recommend that Voice over Internet Protocol implementations in wireless mesh networks with some mobile nodes and background traffic be supported by quality of service standards; else it can lead to service level delivery failures.
\end{abstract}

Key words-wireless; mesh; VoIP; ad hoc; QoS; delay; jitter; packet-loss; mobility

\section{INTRODUCTION}

$\mathrm{T}$ his paper presents the analysis of Voice over Internet Protocol (VoIP) applications on wireless mesh networks (WMNs) with some mobile nodes. We have this scenario in mind for a low cost rural wireless network, to provide free internal VoIP calls on mobile devices. The characteristics of WMNs and VoIP applications cause quality of service (QoS) problems. Studies show that WMNs' unique characteristics are achieved by combining features of mobile adhoc networks (MANETs), wireless sensor networks and cellular technologies [7][16]. These result in QoS issues for VoIP implementations. VoIP applications have sensitivity to delay, jitter, and packet loss; and use small packets. Generally WMNs are considered to be a type of MANET. The similarities between the two are in multi-hop nature and node mobility, but the differences are in the use of gateways, traffic flows, node mobility, mobile node roles and device energy constraints [8] [16].

WMNs usually have dynamic and complex topologies. The next hop can change from time to time and service quality may vary based on the speed of a node's movement, distance from other nodes, obstacles and load on mesh nodes. The IEEE 802.11s standard for WMNs considers mesh nodes as part of the network infrastructure, and mesh nodes can be stationary or mobile. In this paper our WMNs are formed only by wireless mesh clients. We are not considering wireless access points and wireless routers. Our designs are a combination WMNs and MANETs. Studies show that WMNs introduce delay, jitter and packet loss, and may cause problems for VoIP applications. Our analysis mainly studies VoIP applications by discovering how this type of traffic is affected in a WMN with mobile nodes and background traffic. We conducted empirical research by running simulation cases and generating VoIP traffic and non-VoIP traffic. Our simulation cases are set up with stationary and mobile nodes.

\section{RELATED WORK}

A WMN is a communications network made up of radio nodes organized in a mesh topology. Wireless mesh networks often consist of mesh clients, mesh routers and gateways. A WMN is a self-healing, self-organizing and fault tolerant network with dynamic topologies and formed by a mix of wireless clients, access points and/or routers. Studies show that real-time traffic in wireless networks requires QoS for prioritization [15]. For this reason the IEEE 802.11e MAC (Media Access Control) protocol was proposed. Research done by [14] confirms that since wireless mesh networks are characterized as multi-hop transmission, the IEEE 802.11e MAC which deals with QoS does not fit the requirements of backhaul networking in WMNs.

Routing protocols play a key role in order to facilitate mesh node discovery and communication. Therefore choice of routing protocol and its characteristics can affect QoS [8]. There are several important factors for choosing a mesh routing protocol like size of network, node mobility and traffic type. Mesh routing protocols are usually classified as proactive, reactive or hybrid. There are many mesh routing protocols, and some are commonly used: B.A.T.M.A.N, DSDV, HSR, IARP, OLSR and DSR [16]. Another routing protocol named GoDRP (God Routing Protocol, or GoD) is also used by simulation software like ns2 (Network Simulator 2) and NCTUns (National Chiao Tung University- network simulator) to calculate routes based on node positioning and signal range without any routing protocol overhead. This type of routing protocol is used to benchmark simulations to the best way a routing protocol can theoretically perform [17].

Prior to implementing VoIP applications, it is important to understand and test existing networks if they can support VoIP applications. Research shows that WMN characteristics and complexities make it challenging to implement VoIP applications, which are mainly due to multi-hop paths and dynamic nature [7].

Today VoIP applications are widely used, but still there is lack of QoS for voice applications in new emerging WMNs, since VoIP applications are exchanging many small packets, which are made of big packet headers and small VoIP payloads. Research confirms that little effort has been dedicated to address and investigate these problems on wireless multihop networks [9][10]. WMN QoS is usually affected by delay, jitter and packet loss. Usually one-way delay of $200 \mathrm{~ms}$, jitter of less than $100 \mathrm{~ms}$ and packet loss of less than $5 \%$ is acceptable in VoIP conversations [2]. Another study by [6] using the 
common G.729 codec with 20 bytes of VoIP payload, at 50 packets per second shows that taking into account the VoIP silence period can increase the utilization by up to $30 \%$. The silence periods are natural in VoIP conversation where no packets are sent.

VoIP traffic characteristics are unique considering packet size, number of packets per second, inter-packet delays and dependencies on the type of codec being used. Studies by [3] and [11] explain that the G.729 VoIP payload can be from 10 to 40 bytes, but the default is 20 bytes. Since VoIP uses RTP (Real-Time Transport Protocol) with a header of 12 bytes, UDP (User Datagram Protocol) with a header of 8 bytes and IP (Internet Protocol) with a header of 20 bytes, it totals 40 bytes of RTP/UDP/IP headers. Now if we add a VoIP payload of 20 bytes, it sums to 60 bytes without the consideration of data link headers. The G.729 codec with 20 bytes of VoIP payload requires that 50 packets be sent per second. The number of packets can change if the VoIP payload increases or decreases, but for a normal calculation we use 50 packets per second to study the VoIP traffic. Also VoIP conversations tend to have speech periods and silence periods. Studies show that if VoIP applications use a voice activity detection mechanism, and during the silence periods voice packets are not sent, it saves about $35 \%$ of the bandwidth for an average volume of 24 simultaneous calls. Research by [19] shows that the VoIP inter packet delay time is usually between $10-30 \mathrm{~ms}$. The inter packet delay (IDP) time can even increase when the back-off algorithms sense that medium is busy.

Identifying VoIP flows in real-time is important for researchers in order to manage network traffic issues, prioritize VoIP flows, reserve bandwidth or block calls for certain destinations. The research by [20] shows that when two persons A and $\mathrm{B}$ talk to each other, their conversation can be modeled in four states: A talking, B talking, both A and B talking, both silent. A Markov 4-state chain can model these states. Another study by [4] shows that VoIP conversations are made of talkspurts (on periods) and silence gaps (off periods) on the G.729 codec, since human conversation also has talk periods and silence periods.

Node mobility can affect the performance of mesh routing protocols and quality of service. Before a mesh routing protocol is selected the node mobility model has to be identified. An empirical study by [5] has compared DSDV with DSR mesh routing protocols. The comparison of these routing protocols were done on four-node mobility models which are Random Waypoint, Random Point Group Mobility, Freeway Mobility and the Manhattan Mobility models. The research results show that DSR performs better than DSDV in high mobility networks, since DSR has a faster route discovery compared to DSDV when the old route is not available.

VoIP critical metrics and factors that affect QoS in a WMN are delay, jitter, packet loss and bandwidth [13] [7]. Besides these there are also hidden factors like mobility, obstacles and weather conditions that affect the link quality [11]. Mobility is also one of the main factors of measuring mesh QoS [1] [13], but it is one of most complicated and challenging factors to measure. In order to measure the QoS factors there are different methods and tools used to conduct tests and perform data collection. Some researchers have developed their own testing and measurement tools and some researchers have used off the shelf tools like Iperf, rude and crude [6], RTP, STG, RTG, STCP, and RTCP.

\section{Methodology}

Referring back to the literature, it was stated that the IEEE 802.11e standard, which addresses quality of service in wireless networks, is designed for single-hop. Since WMNs are of a multi-hop nature, the IEEE 802.11e standard cannot be applied on them. As a result WMNs can be challenging for VoIP implementation by introducing delay, jitter, packet loss and less bandwidth allocation compared to single-hop for VoIP applications [7] [13]. Therefore our research focuses on the answer for the following question:

\section{How are VoIP QoS factors affected by WMN node mobility?}

The related work shows that there hasn't been much work done by other researchers addressing this type of problem in WMNs, especially when the mesh nodes are both mobile and stationary. Also the related work shows that quality of service for VoIP traffic has remained as a problem among the research community and more research is required to investigate and understand how WMNs affect VoIP applications. The related work also shows that researchers are proposing different solutions, but none of the solutions have solved the problem. This is due to the fact that more research is required in order to study and understand WMN effects on VoIP QoS. This paper's aim is to show and characterize the problems that can affect the VoIP implementations. We studied VoIP implementation in three different WMNs scenarios. We are analyzing the QoS critical factors like delay, jitter, packet loss and bandwidth as discussed in related work, and discovering the reasons that affects these factors and why it goes beyond the acceptable limits. We have measured and analyzed the critical QoS factors for VoIP traffic only, and discover if VoIP applications can be successful in WMNs considering VoIP and WMNs' unique type, nature and characteristics.

Since our aim is to discover the problems that VoIP applications confront when the mesh nodes are moving and measuring some quantities like delay, jitter, packet loss, and bandwidth, an empirical study is required in order to help us discover actual affects of WMNs on service quality [5][12] [15][18]. We configured wireless mesh nodes to generate traffic using a single-channel multi-hop mesh network. We collected and measured data and statistics from the source and destination nodes. We investigated the amount of delay that is caused by a number of hops and load, amount of jitter produced as a result of multiple hops and transmission delay, number of packets lost among the nodes and bandwidth used at the each node by VoIP and non-VoIP applications.

The Simulation Software choice for this research is the NCTUns version 6.0 [18]. With this simulation software we are able to design and simulate WMNs. We have designed three WMN topologies and run test cases in order to analyze the VoIP applications behavior considering WMN node mobility in each scenario. We collected data and statistics in order to analyze how VoIP QoS factors are affected by comparing scenarios and traffic profiles. The test cases have been designed in three difference scenarios. Each scenario is tested against two VoIP traffic profiles. Profile one is a simple VoIP conversation between two mesh nodes without any background traffic. Profile two is VoIP traffic along with background traffic like TCP in greedy mode.

The Experimental design in this research is based on a single-channel multi-hop wireless mesh network on the 802.11b IEEE standard deployed among 26 nodes. By using the simulator we have designed three WMNs topologies each with 26 nodes. All the nodes are operating in ad-hoc mode. 
The 26 nodes are covering an area of almost $132248 \mathrm{~m}^{2}$ $(\mathrm{y}=488 \mathrm{~m}, \mathrm{x}=271 \mathrm{~m})$. Mesh nodes are running the GoD routing protocol [17].

A VoIP profile has been scripted to simulate two persons talking to each other using wireless mesh enabled devices. As a test case we simulated a mother talking to her child, where the mother does most of the talking. During the VoIP conversation there are occasions when mother (speaker 1, TABLE I) and child (speaker 2) are both talking at the same time, mother talking and child listening, child talking and mother listening, or both are silent as done by [20]. When speaker 1 talks, the mesh node is sending VoIP traffic to speaker 2, and the traffic will go across the mesh network to reach speaker 2. Mostly when speaker 1 talks speaker 2 listens and vice versa with silence suppression. This model of communication is based on the Markov model described in [20].

TABLE I. SPEAKER 1 CONFIGURATION SCRIPT. SPEAKER 2 VOIP CONFIGURATION SCRIPT IS THE SAME EXCEPT FOR DIFFERENCE IN VALUES

\begin{tabular}{|c|c|c|c|c|c|c|}
\hline \multirow{2}{*}{$\begin{array}{l}\stackrel{n}{Z} \\
\stackrel{0}{\pi} \\
\dot{n} \\
\dot{x}\end{array}$} & \multirow{2}{*}{ 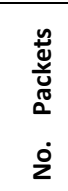 } & \multicolumn{2}{|c|}{$\begin{array}{c}\text { Inter pack- } \\
\text { et delay in } \\
\text { sec }\end{array}$} & \multicolumn{3}{|c|}{$\begin{array}{c}\text { Packet length } \\
\text { in bytes }\end{array}$} \\
\hline & & $\stackrel{\Sigma}{\Sigma}$ & $\sum_{\substack{\pi \\
\Sigma}}^{x}$ & $\stackrel{\infty}{\mathbb{2}}^{\infty 0}$ & $\stackrel{\Sigma}{\Sigma}$ & $\sum_{\substack{\pi \\
\Sigma}}^{x}$ \\
\hline on: & 500 & 0.01 & 0.05 & 70 & 60 & 80 \\
\hline off: & 2 & & & & & \\
\hline on: & 1500 & 0.01 & 0.05 & 70 & 60 & 80 \\
\hline off: & 19 & & & & & \\
\hline on: & 2250 & 0.01 & 0.05 & 70 & 60 & 80 \\
\hline off: & 19 & & & & & \\
\hline on: & 3000 & 0.01 & 0.05 & 70 & 60 & 80 \\
\hline off: & 34 & & & & & \\
\hline on: & 2250 & 0.01 & 0.05 & 70 & 60 & 80 \\
\hline off: & 19 & & & & & \\
\hline on: & 1500 & 0.01 & 0.05 & 70 & 60 & 80 \\
\hline off: & 19 & & & & & \\
\hline on: & 2250 & 0.01 & 0.05 & 70 & 60 & 80 \\
\hline off: & 19 & & & & & \\
\hline on: & 3000 & 0.01 & 0.05 & 70 & 60 & 80 \\
\hline off: & 35 & & & & & \\
\hline on: & 2250 & 0.01 & 0.05 & 70 & 60 & 80 \\
\hline off: & 19 & & & & & \\
\hline on: & 250 & 0.01 & 0.05 & 70 & 60 & 80 \\
\hline off: & 4 & & & & & \\
\hline
\end{tabular}

As discussed in section II, VoIP software usually uses the RTP protocol in order to transport voice traffic over UDP and IP. If we consider VoIP payload size of either 20, 30, 40 bytes and then if we add the RTP/UDP/IP headers to VoIP payload size it will be make 60,70 and 80 bytes respectively. Therefore we used the packet size of 60,70 and 80 bytes to simulate the VoIP payload along with the RTP/UDP/IP headers with 50 packets/sec and IPD of 0.01-0.05ms.

In our VoIP profile, the mother and child conversation flow can be broken up as follows. Mother starts with a short greeting. During the greeting, both mother and child are talking for almost 10 seconds. Here both nodes are talking and generating traffic, therefore both are set to ON mode. Then they pause for 2 seconds, the OFF mode. After that the mother starts talking for 30 seconds, in ON mode, while the child is listening, in OFF mode. This conversation continues for some time with a sequence of $\mathrm{ON}$ and $\mathrm{OFF}$ states and then the mother says goodbye to her child and the child responds with a goodbye and the conversation ends. This conversation takes 562 seconds. In total the mother generates approximately 18250 packets and the child generates 7500 packets for the whole conversation. These numbers of packets are just estimations, and in live applications it can change, since when the voice traffic is packetized it can have varying sizes and this number may increase or decrease depending on the codec being used. Here we are considering the G.729 codec.

Now in order to simulate a human conversation, we have to use a traffic generation tool that can simulate a human VoIP conversation by generating packets with varying sizes, varying inter packet delays, simulating ON and OFF periods. To achieve this we have selected the STG (sent traffic grapher) and RTG (receive traffic grapher) tools. The STG tool is used to send traffic and the RTG is used to receive traffic. STG can be used with several modes like TCP, UDP and configuration which allows us to write a script and translate the human conversation into a form that the STG tool can read from the script and generate the traffic as per our defined VoIP parameters for a human conversation.

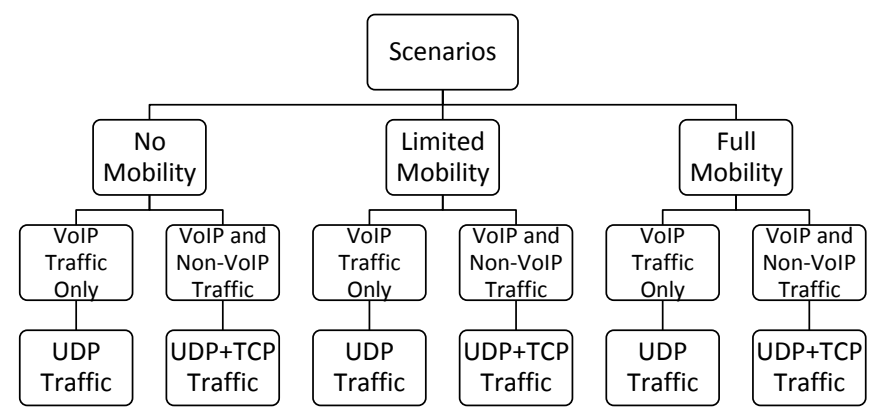

Fig. 1. Simulation Scenarios, with VoIP (UDP) and non-VoIP (TCP)

The non-VoIP profile background traffic is simulated using the STCP and RTCP traffic generation tools. Here we have used a simple TCP greedy traffic mode, where the tool establishes numerous TCP connections between the two communicating nodes and transmits TCP data. This traffic is generated to simulate background traffic. The test cases of VoIP only and VoIP with non-VoIP traffic profiles are simulated in three different scenarios as shown in Fig. 1. Each of the 26 nodes are configured with following traffic generation tool commands. As an example, we show Node 1 and 25 configuration commands for profiles 1 and 2 below. All other nodes are configured the same way in all scenarios.

Node 1 configuration for VoIP-only profile:

stg -i spkrconfig1.cfg -p 4000 1.0.1.25

rtg -u -p 4000 -w pktlog 1 -o thrlog 1

Node 25 Configuration for VoIP-only profile:

stg -i spkrconfig2.cfg - 4000 1.0.1.1

rtg -u -p 4000 -w pktlog25 -o thrlog25

Node 1 configuration for VoIP and non-VoIP profile:

stg -i spkrconfig1.cfg - 40000 1.0.1.25

rtg -u - p 4000 -w pktlog 1 -o thrlog 1

rtcp - p 5000 -w rtcplog1

Node 25 Configuration for VoIP and non-VoIP profile:

stg -i spkrconfig2.cfg - 40000 1.0.1.1

rtg - u - 40000 -w pktlog25 -o thrlog25

stcp - p 5000 1.0.1.1

Scenarios 1, 2 and 3 are WMNs formed by 26 mesh nodes. In scenario 1 all the 26 nodes are stationary and they don't have any movement. Each node is involved in a VoIP conversation with another node. So there are 13 mesh-node peers communicating to each other, shown in TABLE II.

Scenario 2 is designed and configured with 10 nodes moving at a walking speed of $1.3 \mathrm{~m} / \mathrm{sec}$, while the other 16 nodes are stationary. All moving nodes travel along the pre-defined path and return back to their original positions. The aim of this scenario is to discover the effects of mobile and stationary nodes on the VoIP traffic. Scenario 3 is designed and config- 
ured to simulate all the mesh nodes moving in a walking speed of $1.3 \mathrm{~m} / \mathrm{sec}$, shown in Fig. 2. All nodes move to a predefined path (gray lines) and come back to their original position.

TABLE II. SPEAKER $1 \&$ SPEAKER 2 VOIP PEER INFORMATION FOR ALL SCENARIOS. IN TOTAL 13 VOIP PEERS ARE COMMUNICATING.

\begin{tabular}{|l|l|l|}
\hline Peers & Speaker 1 & Speaker 2 \\
\hline 1 & Node 1 & Node 25 \\
\hline 2 & Node 2 & Node 15 \\
\hline 3 & Node 3 & Node 26 \\
\hline 4 & Node 4 & Node 14 \\
\hline 5 & Node 5 & Node 22 \\
\hline 6 & Node 6 & Node 21 \\
\hline 7 & Node 7 & Node 18 \\
\hline 8 & Node 8 & Node 16 \\
\hline 9 & Node 9 & Node 17 \\
\hline 10 & Node 10 & Node 24 \\
\hline 11 & Node 11 & Node 20 \\
\hline 12 & Node 12 & Node 19 \\
\hline 13 & Node 13 & Node 23 \\
\hline
\end{tabular}

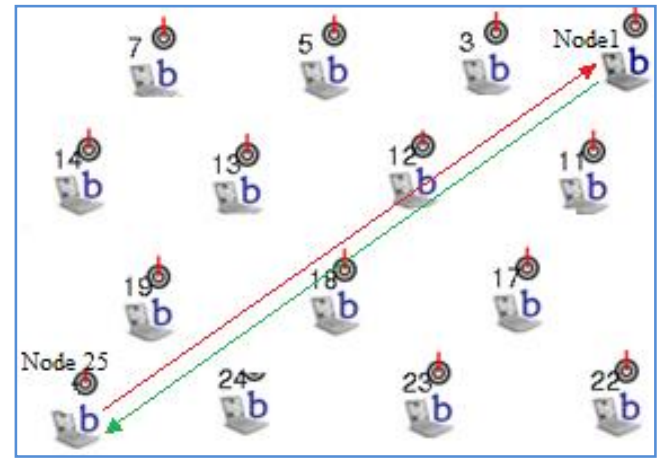

Fig. 2. Scenarios $1,2 \& 3$ have similar screenshots: in scenario 1 all nodes are stationary, in scenario 2 nodes: $2,5,8,9,11,13,16,21,23 \& 25$ are mobile and in scenario 3 all nodes are mobile.

\section{RESULTS}

Analyzing each of the VoIP QoS factors in turn, we can see that delay (Fig. 3) in VoIP only profiles is the lowest, regardless of the node mobility factor. VoIP traffic delay in scenarios with background traffic is mostly higher than $200 \mathrm{~ms}$, only in a few nodes where the VoIP peers are only one hop away, the delay is lower than $200 \mathrm{~ms}$.

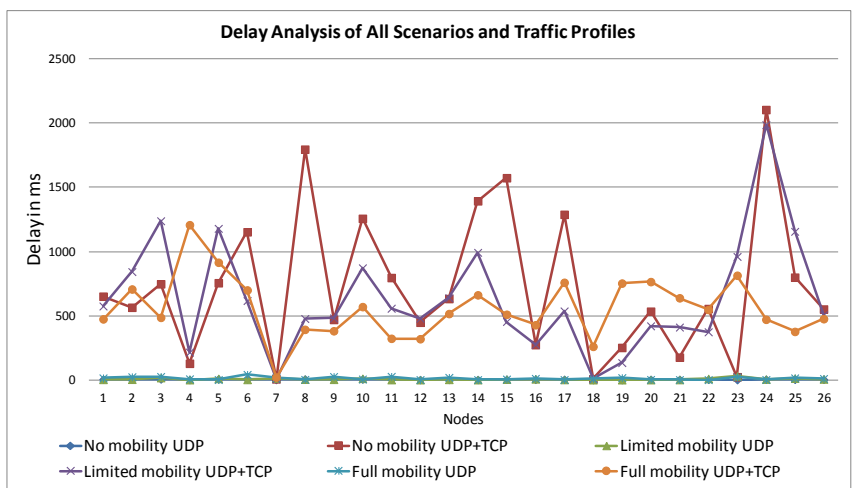

Fig. 3. Scenarios $1,2 \& 3$ VoIP-only profiles have less delay compared to profiles with background traffic.
Jitter analysis (Fig. 4) shows that scenarios with VoIP-only profiles have lower jitter rates, while scenarios with background profile have higher jitter rates. In all scenarios the jitter limit of less than $100 \mathrm{~ms}$ is not crossed.

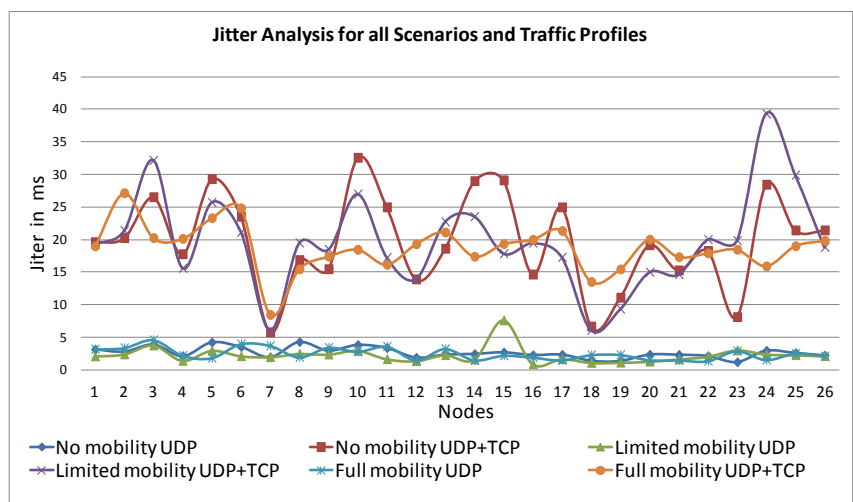

Fig. 4. Scenarios 1, 2 \& 3 VoIP-only profiles have lower jitter rates compared to VoIP profiles with background traffic.

Packet loss analysis (Fig. 5) shows that node mobility increases packet loss. Even scenarios with VoIP-only profiles have packet loss rates above 5\%. Scenarios with background traffic have packet loss reaching up to $80 \%$, only in a few nodes where VoIP peers are one hop away, it is falling below $5 \%$ rate.

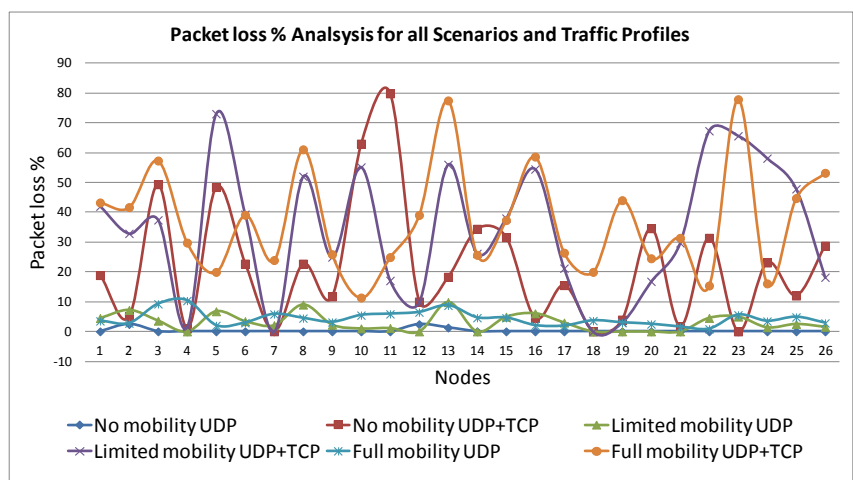

Fig. 5. Nodes Mobility increases packet loss. Scenarios 1, $2 \& 3$ have lower packet loss in VoIP-only profiles.

Throughput analysis (Fig. 6) shows that scenarios with VoIP only profile require $40-45 \mathrm{kbps}$ bandwidth. In scenarios with background traffic, bandwidth allocation and usage are still on the same range, but traffic prioritization and use of priority queues are required, since both types of traffics are using the normal queues.

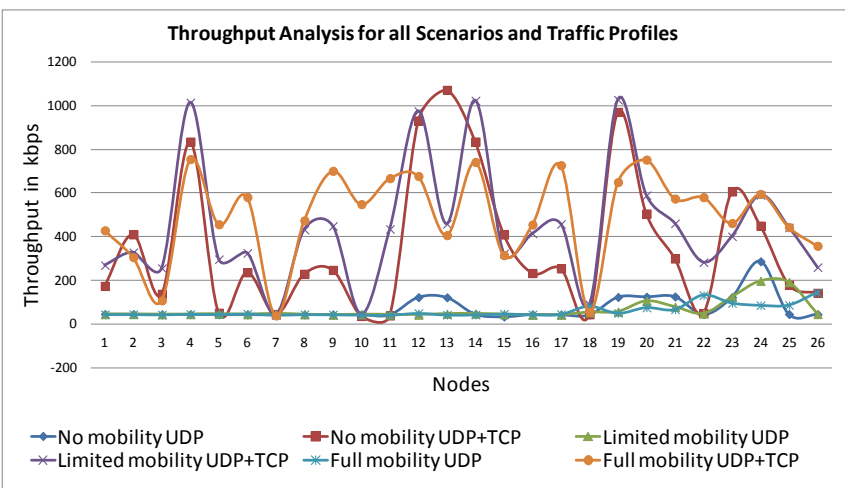

Fig. 6. Scenarios 1, 2 \& 3 use $40-45$ kbps in VoIP-only profiles. 


\section{CONCLUSION AND FUTURE WORK}

Our interest is to deploy low cost VoIP services in a rural wireless mesh network with some mobile nodes. Thus, we need to learn how to address QoS issues. VoIP applications in WMNs, whether nodes are stationary or mobile, can be successful if no background traffic is mixed with VoIP traffic. VoIP implementation with background traffic may not be successful if quality of service is not implemented among mesh nodes. Node mobility can result in more packet loss. If node mobility with a speed of $1.3 \mathrm{~m} / \mathrm{sec}$ causes packet loss, then high node mobility can increase the packet loss to an extent that would make the VoIP implementation unusable. Jitter rate in all three scenarios did not cross the acceptable VoIP jitter limit. If the VoIP enabled wireless mesh nodes happen to be close to each other or only one hop away, the VoIP conversation can run smoothly even if there is background traffic generated or processed by the VoIP enabled nodes.

Recommendations for VoIP implementation over WMNs are as follows. Until QoS standards are supported by WMNs, it is better to create a separate network for VoIP applications, so that other traffic will not mix with VoIP traffic. Routing protocol choice according to the node mobility model has to be made carefully. In WMN design a node's moving path should be defined in such a way that it can move towards its communicating nodes, where possible. If and when possible mesh nodes mobility speed should be kept to minimum.

Limitations of this research involves: simulating one mobility model; running test cases in a simulator, not in a real network; time and hardware constrains in order to simulate a large WMN of more than 100 nodes; simulating test cases using the GoD routing protocol; mesh nodes formation by clients only; usage of one type of VoIP profile; background traffic simulation using TCP in greedy mode; and VoIP traffic parameterization considering the common G.729 codec.

Future work can involve: testing the same scenarios, but using different WMN routing protocols and analyzing the VoIP QoS factors; modifying mesh node mobility speed to a faster speed and study its affects on VoIP QoS factors; modifying mesh node movement path and direction and then analyzing its affects on VoIP QoS factors; increasing the number of mesh nodes and studying its affects on VoIP QoS factors; changing the VoIP profile parameters and characteristics according to other codec types and studying its affects on VoIP QoS factors; configuring the mesh nodes to switch to $\mathrm{ON}$ and OFF states and then studying how the WMN topology changes and how VoIP QoS factors are affected; and adding mesh access points and routers as fixed devices and studying their effects on VoIP QoS.

\section{REFERENCES}

[1] Akunuri, K., Arora, R., \& Guardiola, I., G. (2011). A study of speed aware routing for mobile ad hoc networks. International Journal of Interdisciplinary Telecommunications and Networking, 3, 40-61.

[2] Anand, K., Samrat, G., Samir, R. D., \& Suman, B. (2007). VoIP on wireless meshes: Models, algorithms and evaluation. INFOCOM 2007. 26th IEEE International Conference on Computer Communications, 2036-2044.

[3] Cisco Systems. (2006). Voice over IP - per call bandwidth consumption.

[4] Dang, T. D., Sonkoly, B., \& Molnár, S. (2004). Fractal analysis and modeling of VoIP traffic. Telecommunications Network Strategy and Planning Symposium. NETWORKS 2004, 11th International, $1-8$.

[5] Divecha, B., Abraham, A., Grosan, C., \& Sanyal, S. (2007). Impact of node mobility on MANET routing protocols models. SoftComputing.net, 1-10.

[6] Dragos, N., Samrat, G., Kyungtae, K., \& Rauf, I. (2007). Performance of VoIP in a 802.11 wireless mesh network. Proc. INFOCOM 2006. 25th IEEE International Conference on Computer Communications, Barcelona, Spain. 1-11.

[7] Li, J., Li, Z., \& Mohapatra, p. (2009). Adaptive per hop differentiation for end-to-end delay assurance in multihop wireless networks. Ad Hoc Networks Journal, 7(6): 1169-1182.

[8] Jun, J., \& Sichitiu, M., L. (2008). MRP: Wireless mesh networks routing protocol. Computer Communications Butterworth-Heinemann, 31(7), 1413-1435.

[9] Kaur-Kehal, R., \& Sengupta, J. (2011). A comprehensive review on improving QoS for VoIP in wireless mesh networks. Journal of Global Research in Computer Science, 2, 32-33.

[10] Kim, H., Yun, S., \& Lee, H. (2007). Boosting VoIP capacity of wireless mesh networks through lazy frame aggregation. IEICE Trans. Commun., E90-B, 5, 1283-1285.

[11] Kim, K., \& Shin, K., G. (2006). On accurate measurement of link quality in Multihop wireless mesh networks. Proc. MobiCom '06 Proceedings of the 12th Annual International Conference on Mobile Computing and Networking, 38-49.

[12] Liese, S., Wu, D., \& Mohapatra, P. (2006). Experimental characterization of an $802.11 \mathrm{~b}$ wireless mesh network. Proc. IWCMC '06 Proceedings of the 2006 International Conference on Wireless Communications and Mobile Computing, 587-592.

[13] Mlinarsky, F. (2006).The challenges and importance of testing mesh networks prior to deployment. Rf Design, 29(6), 16-23.

[14] Reddy, B., T., John, J., P., \& Murthy, S. R., C. (2006). Providing MAC QoS for multimedia traffic in $802.11 \mathrm{e}$ based multihop ad hoc wireless networks. Computer Networks, 154-176.

[15] Shao, H. (2004). Multimedia QoS support specified in various wireless network standards. IEEE MultiMedia, 11(4), 75-77.

[16] Waharte, S., Boutaba, R., Iraqi, Y., \& Ishibashi, B. (2006). Routing protocols in wireless mesh networks: Challenges and design considerations. Multimed Tools Appl, (29), 285-303.

[17] Wang, S., Chou, C., \& Lin, C. (2010). The GUI user manual for the NCTUns 6.0 network simulator and emulator Network and System Laboratory, Department of Computer Science, National Chiao Tung University, Taiwan.

[18] Wang, S., \& Lin, Y. (2005). NCTUns network simulation and emulation for wireless resource management. Wireless Communications and Mobile Computing, 5, 899-916.

[19] Wang, X., Chen, S., \& Jajodia, S. (2005). Tracking anonymous peer to peer VoIP calls on the internet. Proc. Computers and Communications Security, 81-91.

[20] Wu, C., Chen, K., Chang, Y., \& Lei, C. (2008). Detecting VoIP traffic based on human conversation patterns. Systems and Applications of IP Telecommunications, 1-8. 